\title{
Editorial: Recent Progress in Surface Electromagnetic Modes
}

\author{
Lin Chen ${ }^{1 *}$, Fei Gao ${ }^{2}$, Wei E. I. Sha ${ }^{3}$ and Zhi Ning Chen ${ }^{4}$ \\ ${ }^{1}$ Shanghai Key Lab of Modern Optical System, University of Shanghai for Science and Technology, Shanghai, China, ${ }^{2}$ Key \\ Laboratory of Advanced Micro/Nano Electronic Devices \& Smart Systems of Zhejiang, College of Information Science and \\ Electronic Engineering, Zhejiang University, Hangzhou, China, ${ }^{3}$ State Key Laboratory of Modern Optical Instrumentation, \\ College of Information Science and Electronic Engineering, Zhejiang University, Hangzhou, China, ${ }^{4}$ Department of Electrical \\ and Computer Engineering, National University of Singapore, Singapore, Singapore
}

Keywords: surface modes, spoof plasmonics, metasurface, local field enhancement, localized surface plasmons

\section{Editorial on the Research Topic}

\section{Recent Progress in Surface Electromagnetic Modes}

Surface electromagnetic (EM) waves, decaying away from an interface between two different materials/structures, have a long history. By combining Maxwell's equations with material properties and boundary conditions, the surface excitations are characterized in terms of their dispersion, spatial profile, and field confinement. The earliest description of Surface EM waves was established in the context of radio waves propagating along the surface of a conductor of finite conductivity $[1,2]$. In parallel to these two well-known contributions, another observation of anomalous intensity drops in spectra when visible light reflects at metallic gratings was also found in the visible domain [3] and later explained by Fano [4]. The concept of surface plasmon polaritons (SPPs) in the visible domain was first proposed by connecting fast electron beams at thin metallic foils [5] with diffraction gratings in the optical domain [6]. Although Ritchie proposed that fast electrons can be used for the excitation of surface plasmons in metal, the requirements of a high electric voltage and a vacuum environment make it impossible for practical applications. In this special issue, Liu et al. give an overview of recent breakthroughs in the low-energy direct excitation of surface plasmons based on an inelastic electron tunneling effect in tunnel junctions. As we have known, one advantage of SPPs is local electric field enhancement with a subwavelength scale, which benefits SPP-based sensors exhibiting high sensitivity and miniaturized size for single molecular sensing applications. The mini-review of Liu and Ma summarizes highly sensitive onedimensional waveguide SPPs sensors in optical range with unique properties of easy integration, the confinement of light to scales one-tenth wavelength, and low cost. Another advantage of SPPs is the breakthrough of the diffraction limit of imaging resolution. The paper by Wang et al. demonstrates that the resolution of ghost imaging can be improved markedly by combining the SPPs structures with the high-frequency information through the structure algorithm. Another paper in this Research Topic by Li et al. demonstrates that the localized buried InGaAs channel n-MOSFETs has a lower leakage current compared to the surface InGaAs channel n-MOSFETs.

On the other hand, research in this field was limited to the visible or near-infrared spectrum for a long time due to the decrease in field penetration into the conductor at lower frequencies. However, as the surface of perfect conductors is textured, electromagnetic surface waves closely resembling SPPs can also be supported at the microwave and terahertz bands [7], which have even been known in the earlier middle of the 20 century $[8,9]$. These spoof SPPs show rich physics and could have a number of important applications. Tang et al. propose broadband and highefficiency conversion between the rectangular waveguide and the planar spoof SPPs at microwave frequencies, which could easily be extended in plasmonic circuits at terahertz frequencies. Besides 
SPPs in the visible range or spoof SPPs at lower frequencies, there are other basic types of plasmonic excitations-localized surface plasmons (LSPs), which are referred to as the localized oscillations of electrons in metal nanoparticles. It is natural to extend the scope of spoof plasmonics to LSPs, known as "spoof LSPs," which have been found in metallic periodically textured sunflower shaped particles or metallic spiral structures both in the microwave [10] and terahertz range [11]. Zhou et al. investigate an electrically two-way Fano resonance switch in the two concentric spoof LSPs by loading a Schottky barrier diode at microwave frequencies. Liao et al. explore novel antenna composed of metallic spiral structure for spoof magnetic LSPs with horizontally polarized omnidirectional radiation. A groundless Spoof SPPs waveguide was applied to feed the antenna to effectively excite the spoof LSPs mode. At millimeter or terahertz frequencies, the transmission coefficient of ultrathin Spoof SPPs waveguide is usually measured by a vector network analyzer using an expanded module with two probe pins placed at ports IN and OUT to introduce and detect millimeter/terahertz signals from the expanded module [12]. The contribution by $\mathrm{Su}$ et al. considers the uncertainty and the impact of imperfect load calibration standard for on wafer calibration method, which benefits the measurement of planar millimeter/terahertz Spoof SPPs circuit.

In parallel to the spoof SPPs concept, another major original concept is metasurface, which exhibits the capacity of EM wavefront manipulation due to the interaction between an EM wave and two-dimensional meta-atom structures [13]. Their advent dramatically expanded the strong wavefront modulation capabilities of photonic materials and devices within the sub-wavelength scale. We note that the corrugated metallic surfaces mentioned above are also examples of metasurfaces with engineered electric responses. Xiong and $\mathrm{Li}$ present a simple free-standing double-layer all metallic metasurface by crossing fractal patterned aluminum foil, with high transmittance and low loss. Li et al. propose a transmission-type fused silica metasurface to manipulate the terahertz wavefront with the function of one/multi-spot focusing and non-diffracting Bessel beam generation. Wang and Zhai perform a microwave reflective angle insensitive circular polarization regulator using a chiral metasurface based on the unit cell having a $\mathrm{z}$-shaped structure. Furthermore, there are several extended contributions with reconfigurable metasurfaces and metadevices. Xiong et al. describe the active control of terahertz wave transmission using Inorganic perovskite quantum dots embedded metasurface. Ding et al. examine a novel periodic metasurface structure with a

\section{REFERENCES}

1. Sommerfeld A. Über die Fortpflanzung electrodynamischer Wellen längs eines Drahtes. Ann Phys und Chemie. (1899) 67:233-90. doi: 10.1002/andp.18993030202

2. Zenneck J. Über die Fortpflanzung ebener elektromagnetischer Wellen längs einer ebenen Leiterfläche und ihre Beziehung zur drahtlosen Telegraphie. Ann d Phys. (1907) 23:846-86. doi: 10.1002/andp.19073281003 through-hole array to improve the gain and radiation of the microstrip antenna. Ni et al. presented a broadband high gain polarization reconfigurable antenna based on metasurfaces. Ji et al. realize a terahertz reflective isolator based on the unique non-reciprocal magneto-plasmonic properties of InSb/dielectric interface to form a resonance cavity between the InSb and metasurface. Chen et al. study a graphene-based terahertz photodetector with metasurface to manipulate the surface EM modes. The photoresponse is enhanced due to the localized EM resonance, resulting in a nearly perfect absorption of the incident terahertz radiation.

Since the seminal works of Ebbesen [14], Pendry [7], and Capasso [13], the study of surface EM modes has been rediscovered and reemerged as an exciting field of research. More recently, the phenomena associated with surface EM modes are rapidly extending and significant work carried out in this field, such as Tamm plasmon-polaritons [15], edge state modes [16], effective SPPs (LSPs) [17], etc.

This Research Topic contains 16 articles devoted to the multifaceted development of ongoing studies in the area of surface EM modes. We add here relevant review articles [18, 19] and hope that this collection will serve as a useful compendium, contributing to growing interest and significant advances in this field that will benefit physicists and engineers in this ongoing field.

The 2021 IEEE APS will be held from December 4 to 10, 2021 in Marina Sands, Singapore. The focus will be state-of-the-art research in antennas, propagation, electromagnetic engineering, and radio science. Information about the Symposium can be found at the website https://2021apsursi.org/.

\section{AUTHOR CONTRIBUTIONS}

All authors listed have made a substantial, direct and intellectual contribution to the work, and approved it for publication.

\section{FUNDING}

This work has received funding in part from the Shanghai Shuguang Program (No. 18SG44).

\section{ACKNOWLEDGMENTS}

The Research Topic editors appreciate the multifaceted contributions of the authors, reviewers, and Frontiers staff who assisted in preparing the manuscripts.

4. Fano U. The theory of anomalous diffraction gratings and of quasi-stationary waves on metallic surfaces (Sommerfeld's waves). J Opt Soc Am. (1941) 31:213-22. doi: 10.1364/JOSA.31.000213

5. Ritchie RH. Plasma losses by fast electrons in thin films. Phys Rev. (1957) 106:874-1. doi: 10.1103/PhysRev.106.874 
6. Ritchie RH, Arakawa ET, Cowan JJ, Hamm RN. Surface-plasmon resonance effect in grating diffraction. Phys Rev Lett. (1968) 21:1530-33. doi: 10.1103/PhysRevLett.21.1530

7. Pendry JB, Martín-Moreno L, Garcia-Vidal FJ. Mimicking surface plasmons with structured surfaces. Science. (2004) 305:8478. doi: $10.1126 /$ science. 1098999

8. Oliner AA, Hessel A. Guided waves on sinusoidally-modulated reactance surface. IRE Trans Antennas Propag. (1959) 7:1615. doi: 10.1109/TAP.1959.1144771

9. Harvey FA. Periodic and guiding structures at microwave frequencies. IRE Trans. Microw Theory Techn. (1960) 8:30-61. doi: 10.1109/tmtt.1960.1124658

10. Pors A, Moreno E, Martin-Moreno L, Pendry JB, Garcia-Vidal FJ. Localized spoof plasmons arise while texturing closed surfaces. Phys Rev Lett. (2012) 108:223905. doi: 10.1103/PhysRevLett.108.223905

11. Chen L, Xu NN. Defect-induced Fano resonances in corrugated plasmonic metamaterials. Adv Opt Mater. (2017) 5:1600960. doi: 10.1002/adom.201600960

12. Chen L, Liao DG, Guo XG, Zhao JY, Zhu YM, Zhuang SL. Terahertz time domain spectroscopy and micro-cavity components for probing samples: a review. Front Inf Technol Electron Eng. (2019) 20:591. doi: 10.1631/FITEE.1800633

13. Yu N, Genevet P, Kats MA, Aieta F, Tetienne JP, Capasso F, et al. Light propagation with phase discontinuities: generalized laws of reflection and refraction. Science. (2011) 334:333-7. doi: 10.1126/science.1210713

14. Ebbesen TW, Lezec HJ, Ghaemi HF, Thio T, Wolff PA. Extraordinary optical transmission through sub-wavelength hole arrays. Nature. (1998) 391:66769. doi: $10.1038 / 35570$
15. Afinogenov BI, Bessonov VO, Soboleva IV, Fedyanin AA. Ultrafast all-optical light control with tamm plasmons in photonic nanostructures. ACS Photonics. (2019) 6:844-50. doi: 10.1021/acsphotonics.8b01792

16. Zeng Y, Chattopadhyay U, Zhu B. Electrically pumped topological laser with valley edge modes. Nature. (2020) 578:24650. doi: 10.1038/s41586-020-1981-x

17. Li Z, Liu LL, Fernandez-Dominguez AT, Shi JF, Gu CQ, Garcia-Vidal FJ, et al. Mimicking localized surface plasmons with structural dispersion. $A d v O p t$ Mater. (2020) 7:10. doi: 10.1002/adom.201900118

18. Gao Z, Wu L, Gao F, Luo Y, Zhang B. Spoof plasmonics: from metamaterial concept to topological description. Adv Mater. (2018) 30:1706683.doi: 10.1002/adma.201706683

19. Zhang X, Xu Q, Xia L, Li Y, Gu J, Tian Z, et al. Terahertz surface plasmonic waves: a review. Adv. Photonics. (2020) 2:014001.doi: 10.1117/1.AP.2.1.014001

Conflict of Interest: The authors declare that the research was conducted in the absence of any commercial or financial relationships that could be construed as a potential conflict of interest.

Copyright (c) 2021 Chen, Gao, Sha and Chen. This is an open-access article distributed under the terms of the Creative Commons Attribution License (CC BY). The use, distribution or reproduction in other forums is permitted, provided the original author(s) and the copyright owner(s) are credited and that the original publication in this journal is cited, in accordance with accepted academic practice. No use, distribution or reproduction is permitted which does not comply with these terms. 